\title{
TEACHING CHINESE AS FOREIGN LANGUAGE IN HIGHER EDUCATION IN CHINA AND SOUTH AFRICA: LECTURERS' VIEWS
}

Yuhua Wang

Tianjin Polytechnic University

Peoples Republic of China

EM Lemmer

College of Education

University of South Africa

As demand for knowledge of the Chinese language has increased, so too have the teaching and learning of Chinese worldwide. This article presents the findings arising from a questionnaire administered to lecturers teaching Chinese as Foreign Language (CFL) in universities in both China and South Africa. CFL programmes offered by universities in China are a popular choice among foreign students who wish to learn Chinese. In addition, the increase in CFL programmes offered by universities in South Africa reflects a growing interest in the Chinese language among South African nationals. The purpose of the questionnaire was to find the differences and similarities between teaching CFL in China and in South Africa, as seen from the lecturers' perspective. It details the research method that was followed and it presents its main findings in tables. The findings consist of a biographical profile, institutional factors, aspects concerning students, teaching methods, and curriculums. The possible reasons for differences and similarities between the two countries are explained.

Key words: Chinese as Foreign Language, China, South Africa, higher education, survey, language pedagogy, lecturers

\section{INTRODUCTION}

The People's Republic of China (PRC) has been a major player on the world political stage for many years by virtue of its size and military capacity, as well as its permanent membership of the United Nations Security Council. Due to internal political change in more recent decades, it has emerged as a major market and trading partner for the West and Africa. As the Chinese economy has grown and more countries have established trading links with China, the Chinese language has become more important worldwide (USA Today, 2007). Today Chinese is one of the six official languages of the United Nations (UN official languages, 2013). For the foreigner interested in China, learning Chinese is regarded as the best way to gain a deeper understanding of China as language is an essential tool to make meaning and interpret social practices. It allows one to access cultural values and beliefs (Kramsch, 1994). International exchanges with China in economics, trade, science, technology, culture, education, art and tourism are more frequent than ever before. As a result, studying Chinese has become increasingly popular and necessary in today's world. 
Thus, Chinese as Foreign Language (CFL) or, alternatively, Mandarin as Foreign Language (MFL) has become a popular subject to study amongst the young in the Western world and in many other parts of the world (BBC News, 2006). Variability in terminology can be observed in the alternative terms (not necessarily synonymous) used by different authors when referring to standard Chinese as acquired by non-native speakers, such as Chinese as a Foreign Language (CFL), Chinese Mandarin as a Foreign Language (CMFL) and Mandarin as a Foreign Language (MFL). Our decision to use CFL as terminology in this article was determined by the wide use of the term in the Anglophone literature on the topic (Wang \& Lemmer, 2014: 415). According to the Confucius Institute Annual Development Report (2013), the interest in CFL is illustrated by increases in enrolment figures, in the demand for teachers of Chinese and in interest in the standardised testing of Chinese in all countries where institutes have been established. The increased interest in the Chinese language and the number of foreign students studying Chinese have resulted in a call for more Chinese language teachers to cope with the demand. In addition, many countries require more Chinese teachers to support local Chinese language education. To satisfy these needs, China has, since 2002, set up a number of non-profit Chinese-language learning institutions overseas. These are now known as Confucius Institutes. These institutes aim to establish a bridge between China and other countries by promoting the teaching and understanding of the Mandarin language and of Chinese culture (Hanban, 2011). By 2013, there were 440 Confucius Institutes and 646 Confucius Classrooms in 120 countries and regions (Confucius Institute Annual Development Report, 2013). The Ministry of Education of the PRC plans to establish 1000 Confucius Institutes by 2020 (Confucius Institute Development Plan, 2012-2020). According to the Hanban Annual Report of 2013, there were 28670 full-time and part-time teachers at the end of 2013, as well as 850000 registered students worldwide (Confucius Institute Annual Development Report, 2013).

Against this background, this article focuses on the differences and similarities of teaching CFL in universities in China and South Africa with special reference to composition of the teaching corps, institutional factors in support of CFL learning, aspects regarding students, CFL teaching methods and CFL curriculum. CFL programmes offered by universities in China are a popular choice among foreign students who wish to learn Chinese. In addition, the increase in CFL programmes offered by universities in South Africa reflects a growing interest in the Chinese language among South African nationals.

\section{OVERVIEW OF CFL IN HIGHER EDUCATION IN SOUTH AFRICA}

South Africa is a multilingual society with eleven official languages. However, English has emerged as the preferred medium of instruction in higher education. The prominence of language diversity is captured in the Language Policy Framework for South African Higher Education which addresses the following issues: languages of instruction in higher education, the future of South African languages as fields of academic study, the promotion of multilingualism in institutional policies, and the study of foreign languages (FLs) (Ministry of Education of South Africa, 2002). Tuition in FLs in higher education is linked to the languages needed to promote the country's cultural, trade and diplomatic relations (Ministry of Education of South Africa, 2002). In this light, the provision of CFL in South African higher education has achieved greater importance in the last 25 years. To date, four major South African universities offer courses in CFL: the University of South Africa (Unisa), Stellenbosch University (SU), Rhodes University (RU) and the University of Cape Town (UCT) (Wang \& Lemmer, 2013). The terms of nomenclature, the terms Mandarin, Mandarin 
Chinese and Chinese are used in the ensuing discussion of South African universities as they appear in the curriculum and marketing material of each respective university.

In 1990, a Centre for Asian Studies was established at Unisa to raise awareness of modern Asia, to initiate research and to promote knowledge of the region. The design and presentation of Mandarin Chinese courses at the institution resulted from the development of this center (Hau-Yoon, 2002: 1). Mandarin Chinese was introduced at first-year level in 1993 and 42 students enrolled for the course. Thanks to consistent enrolment during the first three years (1993-1996), second-year modules were introduced in 1997 and third-year modules in 2002 (Hau-Yoon, 2002: 55). Today, the Centre for Asian Studies is defunct. However, in May 2011, the Centre for Asian Business was established within the Graduate School of Business at Unisa (Unisa, 2011b). Today the Mandarin Chinese Section is located in the Department of Classics and World Languages, which forms part of the College of Human Sciences (Unisa, 2011a). Unisa offers nine modules in Mandarin Chinese and these are structured at three levels. The University, however, does not offer Mandarin Chinese as a major in an independent undergraduate degree. A student can take Mandarin Chinese as a major or ancillary subject for a degree course that falls in the area of humanities and social sciences, economics and management sciences, or theology and religious studies. Students in law or science may take Mandarin Chinese as an ancillary subject. Students may also take Mandarin for non-degree purposes (Unisa, 2013).

At Stellenbosch University (SU), Mandarin was first taught in the Department of Modern Foreign Languages in 2000. China established a Confucius Institute at SU in 2005 and seconded lecturers to the Institute to assist with teaching Chinese (Stellenbosch University, 2009). From 2005, the Department began to offer a full undergraduate degree in Mandarin. In 2007, with the approval of the application for the Hanyu Shuiping Kaoshi (HSK), which is the standardised test in Chinese Proficiency designed by the Hanban (Chinese National Office for Teaching Chinese as a Foreign Language), the Mandarin Section at SU hosted the first HSK test centre in Sub-Saharan Africa (Stellenbosch University, 2011). An important development at SU has been the introduction of an Honours course in Mandarin from 2012 (Zhao, 2012). SU is the first South African university to offer a higher degree in Mandarin.

Since 2009, Rhodes University (RU) has offered Chinese as a one-year credit-bearing course in the School of Languages. This general practical language course is designed for students whose mother tongue is not Chinese. Due to sustained enrolment figures, Chinese Two (second-year module) and Chinese Three (third-year module) have been introduced (Rhodes University, 2012). The purpose of the courses on all three levels is to help students to understand the background linguistic and cultural information and serve as demonstrations of language use (Rhodes University, 2014).

Chinese language tuition was officially launched in January 2010 at the University of Cape Town (UCT). It offers Mandarin as both first-year and second-year courses (UCT, 2013). A Confucius Institute has also been established at UCT. The centre is aimed at promoting the learning of the Chinese language and culture, fostering a broader and more informed understanding of China (both in the Cape Town area and across South Africa), and at strengthening educational and cultural exchanges and friendly cooperation between China and South Africa. 


\section{CFL IN HIGHER EDUCATION IN CHINA}

Teaching CFL in modern China began in 1950, when Tsinghua University set up a Chinese language-training course for international students from Eastern Europe. Since 1978, and due to the opening up policy adopted by China, as well as to the overall position of that country, the drive to teach CFL has been developed vigorously. In July 1987, with the approval of the State Council, the State Leading Group for Teaching CFL was established (Lu \& Zhao, 2011: 117-119). In 1986, the Degree Committee under the State Council accorded the Beijing Language and Culture University (BLCU) and Peking University (and others) the licence to be the first universities to grant MA degrees to foreign Chinese students. In 1998, BLCU began offering doctoral courses in teaching CFL to foreign graduates ( $\mathrm{Lu} \&$ Zhao, 2011: 118). To ensure the quality of teaching CFL teachers, the Guidelines for Teacher Qualification Evaluation of Teaching CFL were promulgated in 1990 to initiate a teacherqualification certificate system for teaching CFL. At present, there are more than 400 universities and colleges offering various Chinese programmes to foreign learners and with total annual enrolment figures of around 50000 (Lu \& Zhao, 2011: 118). In addition to the traditional preparatory programme, undergraduate, graduate and intensive short-term Chinese courses are available.

\section{METHOD}

The main research question was formulated as follows: What are the differences and similarities between teaching CFL at universities in China and South Africa from the lecturers' point of view? This question was addressed by using a cross-sectional survey research design in which data were gathered by a researcher-designed questionnaire administered to lecturers of CFL at three selected universities in China and all four universities offering CFL in South Africa (Unisa, SU, RU and UCT). Selection of the universities in China was done by purposeful and convenience sampling. Information-rich cases for in-depth study were chosen in such a way that the researcher could access them with minimum difficulty (Arthur, Waring, Coe \& Hedges, 2012: 170). The Chinese institutions were as follows: Beijing Language and Culture University (BLCU), a top language university; Nankai University (NU) in Tianjin, a base university offering CFL; and Tianjin University of Science and Technology (TUST) in Tianjin, a general university offering CFL. In South Africa, all the universities offering CFL were chosen as a comprehensive sample (Mertens, 2010: 318, 370).

The questionnaire comprising seven sections (98 closed questions and 1 open question) was compiled and piloted in both countries to ensure the best possible question formulation. Appropriate changes were subsequently effected to the survey questionnaire. The final questionnaire was available in English and Chinese and was distributed by e-mail and in hard copy. The questionnaire's seven sections were as follows: Section A dealt with biographical data (8 items); Section B dealt with institutional factors (19 items); Section C dealt with lecturers' perceptions of CFL students (23 items); Section D dealt with teaching methods (23 items); Section E dealt with curriculum (17 items); and Section $\mathrm{G}$ dealt with degree purposes for honours and postgraduate students ( 8 items) (this article will not present the outcomes of Section G); Section F was an open question. Sections A to G comprised closed items, in which subjects were chosen from predetermined responses (Koshy, 2005: 87; Johnson, 2008: 94-95; McMillan \& Schumacher, 2006: 197). Ethical requirements were met in the covering letter, which stipulated the identity of the chief researcher, the purpose of the study, the 
protection afforded the respondents by keeping identities confidential, the right to withdraw, instructions for completion, opportunity to obtain results and thanks.

\section{Selection of respondents in China}

Non-probability sampling involves using whatever respondents are available to the researcher. This method was used in the current research. Forty-eight teaching staff were approached as part of the survey and 46 of them completed the questionnaire $(\mathrm{N}=46)$. At BLCU, 30 teaching staff were selected with the help of a gatekeeper and 28 completed the questionnaire. NU employs 51 teaching CFL teaching staff. On the day of data collection, 11 staff were present (15 questionnaires had been prepared) and all present completed the questionnaire $(\mathrm{N}=11)$. At TUST, all seven teaching staff completed the questionnaire $(\mathrm{N}=7)$. Questionnaires were distributed personally during the chief researcher's dedicated study visit to China. The response rate was 88 per cent.

\section{South Africa}

Comprehensive non-probability sampling was used to select eight teaching staff as respondents in South Africa (volunteers were not included). This constitutes the total teaching corps of all four universities combined and six participants completed the questionnaire $(\mathrm{N}=6)$. The respondents were distributed as follows: two were from Unisa, two were from SU, one from RU and one from UCT. Questionnaires were distributed by e-mail and in hard copy. Only respondents from SU completed section G (16 questions) as it was the only university in the country that offered an honours course in Mandarin. The response rate was 75 per cent.

The questionnaire responses were analysed by an expert statistician who used the Statistical Analysis Software package, SAS version 8.0 (SAS 2004). Internal consistency reliability for the questionnaire items was established statistically by the Cronbach alpha. In all cases, a Cronbach alpha coefficient of approximately 0.6 or greater was obtained indicating the internal consistency of questionnaire items (Wang, 2014). The very small number of MFL teaching staff at the South African institutions was an obvious limitation in making statistical comparisons. However, this was considered in the analysis. The responses in the data set were not enough to do exploratory factor analysis to validate the constructs. Logical groups were therefore tested by item analysis for reliability. Item analysis was done to assess the reliability of the different dimensions or constructs in the questionnaire via Cronbach's alpha values. Finally, the study was exploratory and descriptive in nature and no attempt has been made to generalize findings.

\section{RESULTS}

This article presents only the results of questionnaire sections A, B, C, D and E due to space constraints. In all tables, percentages have been rounded off to the first decimal point. Only significant tables are presented to conserve space. A general narrative is used for the discussion of the respondents' biographical profile as contained in section A of the questionnaire. 


\section{Section A: Respondents' biographical profile}

The results of the respondents' biographical profile in section A indicated no statistically significant difference in terms of gender, age, academic qualification and teaching experience. For both countries, female CFL lecturer representation is higher than male representation. Most respondents' age fell in the 21-50 age group in both countries and most respondents had a master's degree and more than five years teaching experience. However, the item dealing with the type of employment of CFL lecturers showed a statistically significant difference between the respondents in the two countries. More lecturers are employed permanently in China due to the long history and well-established nature of CFL provision. In South Africa, more lecturers are employed on a fixed-term or contract basis. This can be partly explained by the secondment of lecturers by the Hanban to South African institutions for a limited period of time as part of China's diffusion policy with regard to CFL - an arrangement that fills the gap in the very small pool of suitably qualified academics in South Africa.

\section{Section B: Perceptions of institutional factors}

Table 1 illustrates respondent perceptions of institutional factors for both countries.

Table 1 Agreement rating frequencies and associated row percentage for statements of institutional factor for CFL by country

\begin{tabular}{|c|c|c|c|c|c|c|c|c|c|c|c|c|}
\hline \multirow{3}{*}{\begin{tabular}{|l|} 
Items \\
Frequency \\
Row \%
\end{tabular}} & \multicolumn{10}{|c|}{ Agreement rating } & \multirow{2}{*}{\multicolumn{2}{|c|}{ Total }} \\
\hline & \multicolumn{2}{|c|}{ Disagree ++} & \multicolumn{2}{|c|}{ Disagree } & \multicolumn{2}{|c|}{ Neutral } & \multicolumn{2}{|c|}{ Agree } & \multicolumn{2}{|c|}{ Agree++ } & & \\
\hline & $\stackrel{\Xi}{\Xi}$ & $\underset{\sim}{\mathbb{s}}$ & : & $\underset{\sim}{\mathscr{S}}$ & : & $\underset{\sim}{~}$ & $\stackrel{\Xi}{\Xi}$ & 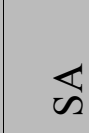 & 芵 & $\underset{\sim}{\mathbb{S}}$ & $\stackrel{\overparen{Z}}{\Xi}$ & $\underset{\sim}{\mathbb{S}}$ \\
\hline $\begin{array}{l}\text { Q1. Stable fund } \\
\text { CFL teaching s }\end{array}$ & $\begin{array}{c}1 \\
2.2\end{array}$ & $\begin{array}{c}2 \\
33.3\end{array}$ & $\begin{array}{c}3 \\
6.5\end{array}$ & $\begin{array}{c}1 \\
16.7\end{array}$ & $\begin{array}{c}15 \\
32.6\end{array}$ & $\begin{array}{c}2 \\
33.3\end{array}$ & $\begin{array}{c}15 \\
32.6\end{array}$ & $\begin{array}{l}0 \\
0\end{array}$ & $\begin{array}{c}12 \\
26.1\end{array}$ & $\begin{array}{c}1 \\
16.7\end{array}$ & 46 & 6 \\
\hline $\begin{array}{l}\text { Q2. Market drive, } \\
\text { recruit CFL studeI }\end{array}$ & $\begin{array}{c}2 \\
4.4\end{array}$ & $\begin{array}{c}1 \\
16.7\end{array}$ & $\begin{array}{c}3 \\
6.5\end{array}$ & $\begin{array}{l}0 \\
0\end{array}$ & $\begin{array}{c}12 \\
26.1\end{array}$ & $\begin{array}{c}4 \\
66.7\end{array}$ & $\begin{array}{c}18 \\
39.1\end{array}$ & $\begin{array}{c}1 \\
16.7\end{array}$ & $\begin{array}{c}11 \\
23.9\end{array}$ & $\begin{array}{l}0 \\
0\end{array}$ & 46 & 6 \\
\hline $\begin{array}{l}\text { Q3. CFL Hanban } \\
\text { subsidy }\end{array}$ & $\begin{array}{c}9 \\
21.4\end{array}$ & $\begin{array}{l}0 \\
0\end{array}$ & $\begin{array}{c}8 \\
19.1\end{array}$ & $\begin{array}{c}1 \\
16.7\end{array}$ & $\begin{array}{c}12 \\
28.6\end{array}$ & $\begin{array}{l}2 \\
33.3\end{array}$ & $\begin{array}{c}9 \\
21.4\end{array}$ & $\begin{array}{c}1 \\
16.7\end{array}$ & $\begin{array}{c}4 \\
9.5\end{array}$ & $\begin{array}{c}2 \\
33.3\end{array}$ & 42 & 6 \\
\hline $\begin{array}{l}\text { Q4. Sufficient staff to } \\
\text { teach CFL }\end{array}$ & $\begin{array}{c}3 \\
6.5\end{array}$ & $\begin{array}{c}1 \\
16.7\end{array}$ & $\begin{array}{c}3 \\
6.5\end{array}$ & $\begin{array}{c}2 \\
33.3\end{array}$ & $\begin{array}{c}5 \\
10.9\end{array}$ & $\begin{array}{c}1 \\
16.7\end{array}$ & \begin{tabular}{|c|}
16 \\
34.8
\end{tabular} & $\begin{array}{c}2 \\
33.3\end{array}$ & $\begin{array}{c}19 \\
41.3\end{array}$ & $\begin{array}{l}0 \\
0\end{array}$ & 46 & 6 \\
\hline $\begin{array}{l}\text { Q5. Teaching staff } \\
\text { adequately qualified }\end{array}$ & $\begin{array}{c}1 \\
2.2\end{array}$ & $\begin{array}{c}1 \\
16.7\end{array}$ & $\begin{array}{c}3 \\
6.5 \\
\end{array}$ & $\begin{array}{c}3 \\
50\end{array}$ & $\begin{array}{c}8 \\
17.4\end{array}$ & $\begin{array}{l}0 \\
0\end{array}$ & $\begin{array}{c}15 \\
32.6\end{array}$ & $\begin{array}{c}1 \\
16.7\end{array}$ & $\begin{array}{c}19 \\
41.3 \\
\end{array}$ & $\begin{array}{c}1 \\
16.7\end{array}$ & 46 & 6 \\
\hline $\begin{array}{l}\text { Q6. Sufficient admin } \\
\text { staff for CFL }\end{array}$ & $\begin{array}{c}3 \\
6.5\end{array}$ & $\begin{array}{c}3 \\
50\end{array}$ & $\begin{array}{c}1 \\
2.2\end{array}$ & $\begin{array}{c}1 \\
16.7\end{array}$ & $\begin{array}{c}9 \\
19.6\end{array}$ & $\begin{array}{l}0 \\
0\end{array}$ & $\begin{array}{c}19 \\
41.3\end{array}$ & $\begin{array}{c}2 \\
33.3\end{array}$ & $\begin{array}{c}14 \\
30.4\end{array}$ & $\begin{array}{l}0 \\
0\end{array}$ & 46 & 6 \\
\hline $\begin{array}{l}\text { Q7. Special } \\
\text { accommodation CFL } \\
\text { students }\end{array}$ & $\begin{array}{l}0 \\
0\end{array}$ & $\begin{array}{c}4 \\
66.7\end{array}$ & $\begin{array}{c}3 \\
6.5\end{array}$ & $\begin{array}{c}2 \\
33.3\end{array}$ & $\begin{array}{c}5 \\
10.9\end{array}$ & $\begin{array}{l}0 \\
0\end{array}$ & $\begin{array}{c}16 \\
34.8\end{array}$ & $\begin{array}{l}0 \\
0\end{array}$ & $\begin{array}{c}22 \\
47.8\end{array}$ & $\begin{array}{l}0 \\
0\end{array}$ & 46 & 6 \\
\hline
\end{tabular}




\begin{tabular}{|c|c|c|c|c|c|c|c|c|c|c|c|c|}
\hline $\begin{array}{l}\text { Q8. Canteen, diet CFL } \\
\text { students }\end{array}$ & $\begin{array}{c}3 \\
6.5\end{array}$ & $\begin{array}{c}4 \\
66.7\end{array}$ & $\begin{array}{c}7 \\
15.2\end{array}$ & $\begin{array}{c}2 \\
33.3\end{array}$ & $\begin{array}{c}3 \\
6.5\end{array}$ & $\begin{array}{l}0 \\
0\end{array}$ & $\begin{array}{c}22 \\
47.8\end{array}$ & $\begin{array}{l}0 \\
0\end{array}$ & $\begin{array}{c}11 \\
23.9\end{array}$ & $\begin{array}{l}0 \\
0\end{array}$ & 46 & 6 \\
\hline $\begin{array}{l}\text { Q9. Special bursaries } \\
\text { CFL students }\end{array}$ & $\begin{array}{c}1 \\
2.2\end{array}$ & $\begin{array}{c}3 \\
50\end{array}$ & $\begin{array}{c}3 \\
6.5\end{array}$ & $\begin{array}{c}2 \\
33.3\end{array}$ & $\begin{array}{c}5 \\
10.9\end{array}$ & $\begin{array}{l}0 \\
0\end{array}$ & $\begin{array}{c}15 \\
32.6\end{array}$ & $\begin{array}{c}1 \\
16.7\end{array}$ & $\begin{array}{c}22 \\
47.8\end{array}$ & $\begin{array}{l}0 \\
0\end{array}$ & 46 & 6 \\
\hline $\begin{array}{l}\text { Q10. CFL courses: } \\
\text { elementary, } \\
\text { intermediate, advanced }\end{array}$ & $\begin{array}{c}1 \\
2.2\end{array}$ & $\begin{array}{c}1 \\
16.7\end{array}$ & $\begin{array}{l}0 \\
0\end{array}$ & $\begin{array}{l}0 \\
0\end{array}$ & $\begin{array}{c}1 \\
2.2\end{array}$ & $\begin{array}{l}0 \\
0\end{array}$ & $\begin{array}{c}13 \\
28.3\end{array}$ & $\begin{array}{c}3 \\
50\end{array}$ & $\begin{array}{c}31 \\
67.4\end{array}$ & $\begin{array}{c}2 \\
33.3\end{array}$ & 46 & 6 \\
\hline Q11. & $\begin{array}{c}1 \\
2.2 \\
\end{array}$ & $\begin{array}{c}1 \\
16.7\end{array}$ & $\begin{array}{c}9 \\
19.6\end{array}$ & $\begin{array}{c}1 \\
16.7\end{array}$ & $\begin{array}{c}6 \\
13.0\end{array}$ & $\begin{array}{c}4 \\
66.6 \\
\end{array}$ & $\begin{array}{l}17 \\
37\end{array}$ & $\begin{array}{l}0 \\
0\end{array}$ & $\begin{array}{c}13 \\
28.3\end{array}$ & $\begin{array}{l}0 \\
0\end{array}$ & 46 & 6 \\
\hline $\begin{array}{l}\text { Q12. Language } \\
\text { laboratory for CFL } \\
\text { students }\end{array}$ & $\begin{array}{c}1 \\
2.3\end{array}$ & $\begin{array}{c}2 \\
33.3\end{array}$ & $\begin{array}{c}6 \\
13.6\end{array}$ & $\begin{array}{c}2 \\
33.3\end{array}$ & $\begin{array}{c}9 \\
20.5\end{array}$ & $\begin{array}{l}0 \\
0\end{array}$ & $\begin{array}{c}15 \\
34.1\end{array}$ & $\begin{array}{c}2 \\
33.3\end{array}$ & $\begin{array}{c}13 \\
29.5\end{array}$ & $\begin{array}{l}0 \\
0\end{array}$ & 44 & 6 \\
\hline $\begin{array}{l}\text { Q13. Large demand } \\
\text { CFL teaching }\end{array}$ & $\begin{array}{c}2 \\
4.4\end{array}$ & $\begin{array}{c}2 \\
33.3 \\
\end{array}$ & $\begin{array}{c}4 \\
8.7\end{array}$ & $\begin{array}{c}1 \\
16.7\end{array}$ & $\begin{array}{c}11 \\
23.9\end{array}$ & $\begin{array}{c}3 \\
50\end{array}$ & $\begin{array}{c}16 \\
34.8\end{array}$ & $\begin{array}{l}0 \\
0\end{array}$ & $\begin{array}{c}13 \\
28.3\end{array}$ & $\begin{array}{l}0 \\
0\end{array}$ & 46 & 6 \\
\hline $\begin{array}{l}\text { Q14. CFL students } \\
\text { increasing }\end{array}$ & $\begin{array}{c}2 \\
4.4\end{array}$ & $\begin{array}{c}1 \\
16.7\end{array}$ & $\begin{array}{c}6 \\
13.1\end{array}$ & $\begin{array}{c}2 \\
33.3\end{array}$ & $\begin{array}{c}16 \\
34.8\end{array}$ & $\begin{array}{c}2 \\
33.3\end{array}$ & $\begin{array}{l}17 \\
37\end{array}$ & $\begin{array}{c}1 \\
16.7\end{array}$ & $\begin{array}{c}5 \\
10.7\end{array}$ & $\begin{array}{l}0 \\
0\end{array}$ & 46 & 6 \\
\hline $\begin{array}{l}\text { Q15. Positive } \\
\text { environment re CFL } \\
\text { conversational skills }\end{array}$ & $\begin{array}{c}1 \\
2.2\end{array}$ & $\begin{array}{c}2 \\
33.3\end{array}$ & $2.2^{1}$ & $\begin{array}{c}3 \\
50\end{array}$ & $\begin{array}{c}11 \\
23.9\end{array}$ & $\begin{array}{c}1 \\
16.7\end{array}$ & $\begin{array}{c}16 \\
34.8\end{array}$ & $\begin{array}{l}0 \\
0\end{array}$ & $\begin{array}{l}17 \\
37\end{array}$ & $\begin{array}{l}0 \\
0\end{array}$ & 46 & 6 \\
\hline $\begin{array}{l}\text { Q16. Sufficient CFL } \\
\text { teaching hours on } \\
\text { timetable }\end{array}$ & $\begin{array}{c}1 \\
2.2\end{array}$ & $\begin{array}{c}3 \\
50\end{array}$ & $\begin{array}{l}0 \\
0\end{array}$ & $\begin{array}{l}0 \\
0\end{array}$ & $\begin{array}{c}4 \\
8.7\end{array}$ & $\begin{array}{c}3 \\
50\end{array}$ & $\begin{array}{l}18 \\
39.1\end{array}$ & $\begin{array}{l}0 \\
0\end{array}$ & $\begin{array}{l}23 \\
50\end{array}$ & $\begin{array}{l}0 \\
0\end{array}$ & 46 & 6 \\
\hline
\end{tabular}

Table 1 shows that Chinese lecturers held statistically significantly more positive perceptions on the institutional infrastructure and support for CFL than South African respondents did. The Chinese lecturers believed that they have stable funding for CFL teaching support with $58.7 \%$ agreeing or strongly agreeing. Among South African lecturers, 50\% disagreed or strongly disagreed with this statement. Among Chinese respondents, $63 \%$ agreed or strongly agreed that a strong marketing drive was in place to recruit CFL students. Among South African lecturers, $66.7 \%$ gave neutral answers here. Most of the Chinese lecturers agreed that they had sufficient staff to teach CFL students $(76.1 \%)$ and that the teaching staff were adequately qualified (73.9\%). Contrary to this, half of the South African lecturers disagreed that they had sufficient staff to teach CFL students and $76.7 \%$ of respondents disagreed that the teaching staff were adequately qualified. The two countries' lecturers also held very contrary attitudes in respect of questions 7, 8 and 9 in section B of the questionnaire. Most of the Chinese lecturers agreed that special accommodations were in place for CFL students (82.6\%), including special canteens $(71.7 \%)$ catering to the diets of CFL students and special bursaries for CFL students $(80.4 \%)$. All the South African lecturers disagreed that special accommodations and/or canteens $(100 \%)$ were in place for CFL students. Most South African lecturers $(83.3 \%)$ did not think that special bursaries were available for CFL students. Generally, institutions in China had language laboratories for CFL students (63.6\% agreed), but South African institutions did not (66.6\% disagreed). Many lecturers in China $(65.3 \%)$ agreed that the CFL courses were highly valued, but most of the South African lecturers $(66.6 \%)$ returned neutral responses. Neutral responses may suggest uncertainty around the issue of how much CFL courses were valued. In South Africa, many (63.1\%) Chinese lecturers responded that there was a large demand for CFL teaching, but half of the South African lecturers returned neutral answers and half disagreed. Other contrasting answers 
appeared in questions 15 and 16. Most of the Chinese lecturers $(71.8 \%)$ agreed that they had a positive environment in which CFL students could practise conversational skills outside of the classroom, but $83.3 \%$ of the South African lecturers disagreed. With regard to teaching hours, $89.1 \%$ of the Chinese lecturers felt that they had sufficient teaching hours on their timetables, but half $(50 \%)$ of the South African lecturers disagreed while the other half returned neutral responses. In addition, the South African lecturers returned a more positive response in respect of the Hanban subsidy than lecturers in China did. Half of the South African lecturers agreed that the university was subsidised by Hanban, but only $30.9 \%$ lecturers in China agreed with this statement.

However, the lecturers in both countries returned similar responses to a few of the questions. Both lecturers in China (95.7\%) and in South Africa (83.3\%) agreed that CFL should be offered at elementary, intermediate and advanced levels. Although less than half of the lecturers $(47.7 \%)$ in China believed that CFL student numbers were growing, $34.8 \%$ still returned neutral answers - a figure similar to South African lecturers' neutral responses $(33.3 \%)$.

The above results (Table 1: Agreement rating frequencies and associated row percentage for statements of institutional factor for CFL by country) call for explanatory comment, which is presented in this paragraph. Compared to South Africa, China has a long history of CFL teaching. Accordingly, the CFL system is mature and well established. Facilities such as language laboratories are very common in the language faculties of higher education institutions. Lecturers in China are thus confident and optimistic as regards the demand for CFL and in respect of stable funding. Further, both the teaching staff and administrative staff are sufficiently well qualified in China. CFL in China is not dependent on Hanban but on the individual institutions at which it is presented. CFL is new to South Africa, having only a 20year history at the institution (Unisa) where it has been available the longest. Other institutions have offered CFL for less than 20 years, such as Stellenbosch University (where it has been offered for 14 years), Rhodes University (5 years) and the University of Cape Town (4 years). Thus, in South Africa, CFL provision is developing steadily but slowly. CFL students in China are foreigners (non-Chinese) studying in China (see Introduction) and Chinese institutions provide separate accommodation and canteens to accommodate their cultural preferences and dietary requirements. In addition, bursaries and grants are offered to encourage them in their studies. With regard to teaching hours, CFL teaching is considered to be intensive in China and each university thus offers about 20 hours per week for Chinese language study only. In China, the general environment is optimal for practising conversation. Conversely, in South Africa, Chinese is a small and new foreign language and CFL students study at home outside of the target language environment. For these reasons, South African universities neither provide CFL students with special accommodation or canteens, nor do they offer dedicated bursaries for CFL. The general environment for CFL is very limited in South Africa and the teaching hours for CFL are based on the curriculum for foreign languages in each individual university. Thus, CFL in South Africa cannot compare with intensive CFL study as it is undertaken in China. Of the four universities that offer CFL in South Africa, three are Hanban subsidised (US, RU and UCT). Of the six respondents to the questionnaire in South Africa, four were seconded by Hanban. Thus, South African lecturers strongly agreed that Hanban subsidies were in place. Conversely, in China, each university has autonomy to appoint and remunerate CFL lecturers. 


\section{Section C: Respondent perceptions of cfl students}

Table 2 presents the respondent perceptions of CFL students.

Table 2 Agreement rating frequencies and associated row percentages for statements of perceptions of CFL students

\begin{tabular}{|c|c|c|c|c|c|c|c|c|c|c|c|c|}
\hline \multirow{3}{*}{\begin{tabular}{|l} 
Items \\
Frequency \\
Row \%
\end{tabular}} & \multicolumn{10}{|c|}{ Agreement rating } & \multirow{2}{*}{\multicolumn{2}{|c|}{ Total }} \\
\hline & \multicolumn{2}{|c|}{ Disagree++ } & \multicolumn{2}{|c|}{ Disagree } & \multicolumn{2}{|c|}{ Neutral } & \multicolumn{2}{|r|}{ Agree } & \multicolumn{2}{|c|}{ Agree++ } & & \\
\hline & 节 & 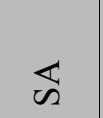 & 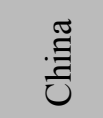 & $\mathbb{n}$ & 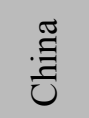 & 倌 & 节 & 岕 & : & 质 & $\stackrel{\Xi}{\Xi}$ & 岕 \\
\hline $\begin{array}{l}\text { Q1. Stable funding for } \\
\text { CFL teaching support }\end{array}$ & $\begin{array}{c}1 \\
2.2\end{array}$ & $\begin{array}{c}2 \\
33.3 \\
\end{array}$ & $\begin{array}{c}3 \\
6.5\end{array}$ & $\begin{array}{c}1 \\
16.7\end{array}$ & $\begin{array}{c}15 \\
32.6\end{array}$ & \begin{tabular}{|c|}
2 \\
33.3 \\
\end{tabular} & $\begin{array}{c}15 \\
32.6\end{array}$ & $\begin{array}{l}0 \\
0\end{array}$ & $\begin{array}{c}12 \\
26.1\end{array}$ & $\begin{array}{c}1 \\
16.7\end{array}$ & 46 & 6 \\
\hline $\begin{array}{l}\text { Q2. Market drive, recruit } \\
\text { CFL students }\end{array}$ & $\begin{array}{c}2 \\
4.4\end{array}$ & \begin{tabular}{c|}
1 \\
16.7
\end{tabular} & $\begin{array}{c}3 \\
6.5\end{array}$ & $\begin{array}{l}0 \\
0\end{array}$ & $\begin{array}{c}12 \\
26.1\end{array}$ & \begin{tabular}{|c|}
4 \\
66.7 \\
\end{tabular} & $\begin{array}{c}18 \\
39.1\end{array}$ & $\begin{array}{c}1 \\
16.7\end{array}$ & $\begin{array}{c}11 \\
23.9\end{array}$ & $\begin{array}{l}0 \\
0\end{array}$ & 46 & 6 \\
\hline Q3. CFL Hanban subsidy & $\begin{array}{c}9 \\
21.4\end{array}$ & $\begin{array}{l}0 \\
0\end{array}$ & $\begin{array}{c}8 \\
19.1\end{array}$ & $\begin{array}{c}1 \\
16.7\end{array}$ & $\begin{array}{c}12 \\
28.6\end{array}$ & $\begin{array}{l}2 \\
33.3\end{array}$ & $\begin{array}{c}9 \\
21.4\end{array}$ & $\begin{array}{c}1 \\
16.7\end{array}$ & $\begin{array}{c}4 \\
9.5\end{array}$ & $\begin{array}{c}2 \\
33.3\end{array}$ & 42 & 6 \\
\hline $\begin{array}{l}\text { Q4. Sufficient staff to teach } \\
\text { CFL }\end{array}$ & $\begin{array}{c}3 \\
6.5\end{array}$ & \begin{tabular}{c|}
1 \\
16.7
\end{tabular} & $\begin{array}{c}3 \\
6.5\end{array}$ & $\begin{array}{c}2 \\
33.3\end{array}$ & $\begin{array}{c}5 \\
10.9\end{array}$ & \begin{tabular}{c|}
1 \\
16.7
\end{tabular} & $\begin{array}{c}16 \\
34.8\end{array}$ & $\begin{array}{c}2 \\
33.3\end{array}$ & $\begin{array}{c}19 \\
41.3\end{array}$ & $\begin{array}{l}0 \\
0\end{array}$ & 46 & 6 \\
\hline $\begin{array}{l}\text { Q5. Teaching staff } \\
\text { adequately qualified }\end{array}$ & $\begin{array}{c}1 \\
2.2\end{array}$ & \begin{tabular}{c|}
1 \\
16.7
\end{tabular} & $\begin{array}{c}3 \\
6.5\end{array}$ & $\begin{array}{c}3 \\
50\end{array}$ & $\begin{array}{c}8 \\
17.4\end{array}$ & $\begin{array}{l}0 \\
0\end{array}$ & $\begin{array}{c}15 \\
32.6\end{array}$ & $\begin{array}{c}1 \\
16.7\end{array}$ & $\begin{array}{c}19 \\
41.3\end{array}$ & $\begin{array}{c}1 \\
16.7\end{array}$ & 46 & 6 \\
\hline $\begin{array}{l}\text { Q6. Sufficient admin staff } \\
\text { for CFL }\end{array}$ & $\begin{array}{c}3 \\
6.5\end{array}$ & $\begin{array}{c}3 \\
50\end{array}$ & $\begin{array}{c}1 \\
2.2\end{array}$ & $\begin{array}{c}1 \\
16.7\end{array}$ & $\begin{array}{c}9 \\
19.6\end{array}$ & $\begin{array}{l}0 \\
0\end{array}$ & $\begin{array}{c}19 \\
41.3\end{array}$ & $\begin{array}{c}2 \\
33.3\end{array}$ & $\begin{array}{c}14 \\
30.4\end{array}$ & $\begin{array}{l}0 \\
0\end{array}$ & 46 & 6 \\
\hline $\begin{array}{l}\text { Q7. Special accommodation } \\
\text { CFL students }\end{array}$ & $\begin{array}{l}0 \\
0\end{array}$ & \begin{tabular}{c|}
4 \\
66.7
\end{tabular} & $\begin{array}{c}3 \\
6.5\end{array}$ & $\begin{array}{c}2 \\
33.3\end{array}$ & $\begin{array}{c}5 \\
10.9\end{array}$ & $\begin{array}{l}0 \\
0\end{array}$ & $\begin{array}{c}16 \\
34.8\end{array}$ & $\begin{array}{l}0 \\
0\end{array}$ & $\begin{array}{c}22 \\
47.8\end{array}$ & $\begin{array}{l}0 \\
0\end{array}$ & 46 & 6 \\
\hline $\begin{array}{l}\text { Q8. Canteen, diet CFL } \\
\text { students }\end{array}$ & $\begin{array}{c}3 \\
6.5\end{array}$ & \begin{tabular}{|c|}
4 \\
66.7
\end{tabular} & $\begin{array}{c}7 \\
15.2\end{array}$ & $\begin{array}{c}2 \\
33.3\end{array}$ & $\begin{array}{c}3 \\
6.5\end{array}$ & $\begin{array}{l}0 \\
0\end{array}$ & $\begin{array}{c}22 \\
47.8\end{array}$ & $\begin{array}{l}0 \\
0\end{array}$ & $\begin{array}{c}11 \\
23.9\end{array}$ & $\begin{array}{l}0 \\
0\end{array}$ & 46 & 6 \\
\hline $\begin{array}{l}\text { Q9. Special bursaries CFL } \\
\text { students }\end{array}$ & $\begin{array}{c}1 \\
2.2\end{array}$ & $\begin{array}{c}3 \\
50\end{array}$ & $\begin{array}{c}3 \\
6.5\end{array}$ & $\begin{array}{c}2 \\
33.3\end{array}$ & $\begin{array}{c}5 \\
10.9\end{array}$ & $\begin{array}{l}0 \\
0\end{array}$ & $\begin{array}{c}15 \\
32.6\end{array}$ & $\begin{array}{c}1 \\
16.7\end{array}$ & $\begin{array}{c}22 \\
47.8\end{array}$ & $\begin{array}{l}0 \\
0\end{array}$ & 46 & 6 \\
\hline $\begin{array}{l}\text { Q10. CFL courses: } \\
\text { elementary, intermediate, } \\
\text { advanced }\end{array}$ & $\begin{array}{c}1 \\
2.2\end{array}$ & \begin{tabular}{c|}
1 \\
16.7
\end{tabular} & $\begin{array}{l}0 \\
0\end{array}$ & $\begin{array}{l}0 \\
0\end{array}$ & $\begin{array}{c}1 \\
2.2\end{array}$ & $\begin{array}{l}0 \\
0\end{array}$ & $\begin{array}{c}13 \\
28.3\end{array}$ & $\begin{array}{c}3 \\
50\end{array}$ & $\begin{array}{c}31 \\
67.4\end{array}$ & $\begin{array}{c}2 \\
33.3\end{array}$ & 46 & 6 \\
\hline Q11. CFL highly valued & $\begin{array}{c}1 \\
2.2\end{array}$ & \begin{tabular}{c|}
1 \\
16.7
\end{tabular} & $\begin{array}{c}9 \\
19.6\end{array}$ & $\begin{array}{c}1 \\
16.7\end{array}$ & $\begin{array}{c}6 \\
13.0\end{array}$ & \begin{tabular}{|c|}
4 \\
66.6
\end{tabular} & $\begin{array}{l}17 \\
37\end{array}$ & $\begin{array}{l}0 \\
0\end{array}$ & $\begin{array}{c}13 \\
28.3\end{array}$ & $\begin{array}{l}0 \\
0\end{array}$ & 46 & 6 \\
\hline $\begin{array}{l}\text { Q12. Language laboratory } \\
\text { for CFL students }\end{array}$ & $\begin{array}{c}1 \\
2.3\end{array}$ & $\begin{array}{c}2 \\
33.3 \\
\end{array}$ & $\begin{array}{c}6 \\
13.6\end{array}$ & $\begin{array}{c}2 \\
33.3\end{array}$ & $\begin{array}{c}9 \\
20.5\end{array}$ & $\begin{array}{l}0 \\
0\end{array}$ & $\begin{array}{c}15 \\
34.1\end{array}$ & $\begin{array}{c}2 \\
33.3\end{array}$ & $\begin{array}{c}13 \\
29.5\end{array}$ & $\begin{array}{l}0 \\
0\end{array}$ & 44 & 6 \\
\hline
\end{tabular}




\begin{tabular}{|l|c|c|c|c|c|c|c|c|c|c|c|c|}
\hline Q13. Large demand CFL & 2 & 2 & 4 & 1 & 11 & 3 & 16 & 0 & 13 & 0 & 46 & 6 \\
teaching & 4.4 & 33.3 & 8.7 & 16.7 & 23.9 & 50 & 34.8 & 0 & 28.3 & 0 & & \\
\hline Q14. CFL students & 2 & 1 & 6 & 2 & 16 & 2 & 17 & 1 & 5 & 0 & 46 & 6 \\
increasing & 4.4 & 16.7 & 13.1 & 33.3 & 34.8 & 33.3 & 37 & 16.7 & 10.7 & 0 & & \\
\hline Q15. Positive environment & 1 & 2 & 1 & 3 & 11 & 1 & 16 & 0 & 17 & 0 & 46 & 6 \\
re CFL conversational skills & 2.2 & 33.3 & 2.2 & 50 & 23.9 & 16.7 & 34.8 & 0 & 37 & 0 & & \\
\hline Q16. Sufficient CFL & 1 & 3 & 0 & 0 & 4 & 3 & 18 & 0 & 23 & 0 & 46 & 6 \\
teaching hours on timetable & 2.2 & 50 & 0 & 0 & 8.7 & 50 & 39.1 & 0 & 50 & 0 & & \\
\hline
\end{tabular}

According to Table 2, lecturers indicated that CFL students in both countries study full-time and the enrolment of CFL students decreased as courses became more advanced. In addition, CFL students were found to study as many hours as students studying other university courses. Respondents perceived that students in both countries believed Chinese to be a difficult language and found the four tones to be very difficult. The students in both countries had the opportunity to visit or travel in China and to compete in national Chinese language or cultural competitions, but they did not have much opportunity to study traditional Chinese characters. They felt free to ask questions in the classroom and they could easily find some study materials - with the exception of their textbooks.

Half of the respondents in China agreed that the CFL students were mostly speakers of other Asian languages, while $83.3 \%$ of the South African respondents agreed that the CFL students were mostly English speakers. Most Chinese lecturers (60\%) believed that the CFL students enjoyed studying Chinese, while only $33.3 \%$ of South African lecturers agreed that students enjoyed studying. Most lecturers in China (75.6\%) agreed that the CFL students had the opportunity to choose other subject fields related to CFL as part of their degree, but $83.3 \%$ of lecturers in South Africa disagreed. More than half (52.4\%) of the Chinese lecturers felt that outstanding students who have graduated from the degree programme wanted to enrol in a postgraduate degree in Mandarin (e.g. honours, master's or doctorate), but $50 \%$ of the South African lecturers disagreed. Lecturers in both countries (China 37.7\%, South Africa 33.3\%) gave neutral answers in respect of Chinese characters being too difficult and students not liking to study them.

\section{Section D: Teaching method}

Table 3 presents the respondent perceptions of the teaching method used in CFL tuition in both countries.

Table 3 Agreement rating frequencies and associated row percentages for statements in respect of CFL teaching method 


\begin{tabular}{|c|c|c|c|c|c|c|c|c|c|c|c|c|}
\hline \multirow{3}{*}{\begin{tabular}{|l} 
Items \\
Frequency \\
Row \%
\end{tabular}} & \multicolumn{10}{|c|}{ Agreement rating } & \multirow{2}{*}{\multicolumn{2}{|c|}{ Total }} \\
\hline & \multicolumn{2}{|c|}{ Disagree++ } & \multicolumn{2}{|c|}{ Disagree } & \multicolumn{2}{|c|}{ Neutral } & \multicolumn{2}{|c|}{ Agree } & \multicolumn{2}{|c|}{ Agree++ } & & \\
\hline & : & 仿 & 芯 & $\omega$ & 氞 & & 音 & 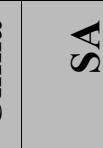 & : & 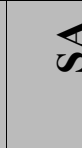 & 莺 & 约 \\
\hline $\begin{array}{l}\text { Q1. CFL } \\
\text { lecturer/student ratio } \\
\text { satisfaction }\end{array}$ & $\begin{array}{c}1 \\
2.2\end{array}$ & $\begin{array}{l}0 \\
0\end{array}$ & $\begin{array}{c}2 \\
4.4\end{array}$ & $\begin{array}{l}0 \\
0\end{array}$ & $\begin{array}{c}10 \\
22.2\end{array}$ & $\begin{array}{c}1 \\
16.7\end{array}$ & $\begin{array}{c}21 \\
46.7\end{array}$ & \begin{tabular}{|c}
4 \\
66.7
\end{tabular} & $\begin{array}{c}11 \\
24.4\end{array}$ & $\begin{array}{l}0 \\
0\end{array}$ & 45 & 5 \\
\hline $\begin{array}{l}\text { Q2. Traditional lecture } \\
\text { method is good for CFL } \\
\text { teaching. }\end{array}$ & $\begin{array}{c}1 \\
2.2\end{array}$ & $\begin{array}{l}0 \\
0\end{array}$ & $\begin{array}{c}2 \\
4.4\end{array}$ & $\begin{array}{c}1 \\
16.7\end{array}$ & $\begin{array}{c}15 \\
32.6\end{array}$ & $\begin{array}{r}3 \\
50\end{array}$ & $\begin{array}{c}22 \\
47.8\end{array}$ & $\begin{array}{c}2 \\
33.3\end{array}$ & $\begin{array}{c}6 \\
13.0\end{array}$ & $\begin{array}{l}0 \\
0\end{array}$ & 46 & 6 \\
\hline $\begin{array}{l}\text { Q3. Memorisation is } \\
\text { essential CFL study. }\end{array}$ & $\begin{array}{c}1 \\
2.2 \\
\end{array}$ & $\begin{array}{l}0 \\
0\end{array}$ & $0^{0}$ & $\begin{array}{l}0 \\
0\end{array}$ & $\begin{array}{c}8 \\
17.4\end{array}$ & $\begin{array}{l}1 \\
16.7 \\
\end{array}$ & $\begin{array}{c}25 \\
54.3\end{array}$ & $\begin{array}{c}5 \\
83.3 \\
\end{array}$ & $\begin{array}{c}12 \\
26.1\end{array}$ & 0 & 46 & 6 \\
\hline $\begin{array}{l}\text { Q4. Students are the } \\
\text { focus of teaching. }\end{array}$ & $\begin{array}{c}2 \\
4.4\end{array}$ & $\begin{array}{l}0 \\
0\end{array}$ & $\begin{array}{c}1 \\
2.2\end{array}$ & $\begin{array}{c}2 \\
33.3\end{array}$ & $\begin{array}{c}3 \\
6.5\end{array}$ & $0^{0}$ & $\begin{array}{c}24 \\
52.2\end{array}$ & $\begin{array}{c}1 \\
16.7\end{array}$ & $\begin{array}{c}16 \\
34.8\end{array}$ & $\begin{array}{c}3 \\
50\end{array}$ & 46 & 6 \\
\hline $\begin{array}{l}\text { Q5. Speak only Chinese } \\
\text { in teaching of CFL. }\end{array}$ & $\begin{array}{c}1 \\
2.2\end{array}$ & $\begin{array}{c}1 \\
16.7\end{array}$ & $\begin{array}{l}0 \\
0\end{array}$ & $\begin{array}{c}3 \\
50\end{array}$ & $\begin{array}{c}6 \\
13.0\end{array}$ & $\begin{array}{c}2 \\
33.3\end{array}$ & $\begin{array}{c}22 \\
47.8\end{array}$ & 0 & $\begin{array}{c}17 \\
37.0\end{array}$ & $\begin{array}{l}0 \\
0\end{array}$ & 46 & 6 \\
\hline $\begin{array}{l}\text { Q6. Speak } \\
\text { Chinese/English in } \\
\text { teaching of CFL }\end{array}$ & $\begin{array}{c}11 \\
25.6\end{array}$ & $\begin{array}{l}0 \\
0\end{array}$ & $\begin{array}{c}15 \\
34.9\end{array}$ & $\begin{array}{l}0 \\
0\end{array}$ & $\begin{array}{c}9 \\
20.9\end{array}$ & $\begin{array}{c}2 \\
33.3\end{array}$ & $\begin{array}{c}6 \\
14.0\end{array}$ & $\begin{array}{c}3 \\
50\end{array}$ & $\begin{array}{c}2 \\
4.7\end{array}$ & $\begin{array}{r}1 \\
16.7\end{array}$ & 43 & 6 \\
\hline $\begin{array}{l}\text { Q7. Use mostly En } \\
\text { in teaching of CFI }\end{array}$ & $\begin{array}{c}35 \\
77.8\end{array}$ & $\begin{array}{c}1 \\
16.7\end{array}$ & $\begin{array}{c}8 \\
17.8\end{array}$ & $\begin{array}{c}2 \\
33.3\end{array}$ & $\begin{array}{c}2 \\
4.4\end{array}$ & $\begin{array}{c}1 \\
16.7\end{array}$ & $\begin{array}{l}0 \\
0\end{array}$ & $\begin{array}{c}1 \\
16.7\end{array}$ & $0^{0}$ & $\begin{array}{l}0 \\
0\end{array}$ & 45 & 6 \\
\hline $\begin{array}{l}\text { Q8. Use same teaching } \\
\text { methods. }\end{array}$ & $\begin{array}{c}12 \\
27.3\end{array}$ & $\begin{array}{c}3 \\
50\end{array}$ & $\begin{array}{c}17 \\
38.6\end{array}$ & $50^{3}$ & $\begin{array}{c}12 \\
27.3\end{array}$ & $\begin{array}{l}0 \\
0\end{array}$ & $\begin{array}{r}3 \\
6.8\end{array}$ & $\begin{array}{l}0 \\
0\end{array}$ & $\begin{array}{l}0 \\
0\end{array}$ & $\begin{array}{l}0 \\
0\end{array}$ & 44 & 6 \\
\hline $\begin{array}{l}\text { Q9. Textbooks are the } \\
\text { basis for teaching. }\end{array}$ & $\begin{array}{c}3 \\
6.5 \\
\end{array}$ & $\begin{array}{l}0 \\
0\end{array}$ & $\begin{array}{c}1 \\
2.2\end{array}$ & $0^{0}$ & $\begin{array}{c}7 \\
15.2\end{array}$ & $\begin{array}{c}1 \\
16.7\end{array}$ & $\begin{array}{c}29 \\
63.0\end{array}$ & $\begin{array}{c}4 \\
66.7\end{array}$ & $\begin{array}{c}6 \\
13.0\end{array}$ & $\begin{array}{r}1 \\
16.7\end{array}$ & 46 & 6 \\
\hline $\begin{array}{l}\text { Q10. Not only textbooks } \\
\text { used to teach. }\end{array}$ & $\begin{array}{c}17 \\
37.8\end{array}$ & $\begin{array}{c}2 \\
33.3\end{array}$ & $\begin{array}{c}20 \\
44.4\end{array}$ & $\begin{array}{c}4 \\
66.7\end{array}$ & $\begin{array}{c}6 \\
13.3\end{array}$ & $\begin{array}{l}0 \\
0\end{array}$ & $\begin{array}{c}2 \\
4.4\end{array}$ & $\begin{array}{l}0 \\
0\end{array}$ & $\begin{array}{l}0 \\
0\end{array}$ & 0 & 45 & 6 \\
\hline $\begin{array}{l}\text { not rely only on } \\
\text { s. }\end{array}$ & $\begin{array}{l}0 \\
0\end{array}$ & $\begin{array}{l}0 \\
0\end{array}$ & $\begin{array}{c}4 \\
9.0\end{array}$ & 0 & $\begin{array}{c}5 \\
11.4 \\
\end{array}$ & $\begin{array}{c}1 \\
20 \\
\end{array}$ & $\begin{array}{c}16 \\
36.4 \\
\end{array}$ & $\begin{array}{c}2 \\
40 \\
\end{array}$ & $\begin{array}{c}19 \\
43.2\end{array}$ & $\begin{array}{l}2 \\
40 \\
\end{array}$ & 44 & 6 \\
\hline $\begin{array}{l}\text { Q12. CFL students } \\
\text { reproduce material } \\
\text { taught. }\end{array}$ & $\begin{array}{c}1 \\
2.2\end{array}$ & $\begin{array}{l}0 \\
0\end{array}$ & $\begin{array}{l}0 \\
0\end{array}$ & $\begin{array}{l}0 \\
0\end{array}$ & $\begin{array}{c}12 \\
26.1\end{array}$ & $\begin{array}{c}1 \\
16.7\end{array}$ & $\begin{array}{c}16 \\
34.8\end{array}$ & $\begin{array}{c}3 \\
50\end{array}$ & $\begin{array}{c}17 \\
37.0\end{array}$ & $\begin{array}{c}2 \\
33.3\end{array}$ & 46 & 6 \\
\hline $\begin{array}{l}\text { Q13. Opportunity to } \\
\text { communicate with } \\
\text { fellow students. }\end{array}$ & $\begin{array}{c}1 \\
2.2\end{array}$ & $\begin{array}{l}0 \\
0\end{array}$ & $\begin{array}{l}0 \\
0\end{array}$ & $\begin{array}{l}0 \\
0\end{array}$ & $\begin{array}{r}4 \\
8.7\end{array}$ & $\begin{array}{c}2 \\
33.3\end{array}$ & $\begin{array}{c}19 \\
41.3\end{array}$ & $\begin{array}{c}3 \\
50\end{array}$ & $\begin{array}{c}21 \\
46.7\end{array}$ & $\begin{array}{r}1 \\
16.7\end{array}$ & 45 & 6 \\
\hline $\begin{array}{l}\text { Q14. Prefer questions } \\
\text { after class, not } \\
\text { interruptions. }\end{array}$ & $\begin{array}{l}11 \\
25\end{array}$ & $\begin{array}{c}1 \\
16.7\end{array}$ & $\begin{array}{l}11 \\
25\end{array}$ & $\begin{array}{c}1 \\
16.7\end{array}$ & $\begin{array}{l}11 \\
25\end{array}$ & $\begin{array}{c}3 \\
50\end{array}$ & $\begin{array}{c}8 \\
18.2\end{array}$ & \begin{tabular}{|c}
1 \\
16.7
\end{tabular} & $\begin{array}{c}3 \\
6.8\end{array}$ & $\begin{array}{l}0 \\
0\end{array}$ & 44 & 6 \\
\hline $\begin{array}{l}\text { Q15. Practice more } \\
\text { important than } \\
\text { explanation. }\end{array}$ & $\begin{array}{l}0 \\
0\end{array}$ & $\begin{array}{l}0 \\
0\end{array}$ & $\begin{array}{r}1 \\
2.2\end{array}$ & $\begin{array}{l}0 \\
0\end{array}$ & $\begin{array}{c}11 \\
24.4\end{array}$ & $\begin{array}{c}2 \\
33.3\end{array}$ & $\begin{array}{c}26 \\
57.8\end{array}$ & \begin{tabular}{|c}
3 \\
50
\end{tabular} & $\begin{array}{c}7 \\
15.6\end{array}$ & $\begin{array}{c}1 \\
16.7\end{array}$ & 45 & 6 \\
\hline $\begin{array}{l}\text { Q16. Listen, speak, } \\
\text { read, write, } \\
\text { understand: goal. }\end{array}$ & $\begin{array}{c}1 \\
2.2\end{array}$ & $\begin{array}{c}1 \\
20\end{array}$ & $\begin{array}{c}3 \\
6.7\end{array}$ & $\begin{array}{l}0 \\
0\end{array}$ & $\begin{array}{c}11 \\
24 . \\
4\end{array}$ & $\begin{array}{c}1 \\
20\end{array}$ & $\begin{array}{l}24 \\
53 . \\
3\end{array}$ & $\begin{array}{l}2 \\
40\end{array}$ & $\begin{array}{l}7 \\
15 \\
5\end{array}$ & $\begin{array}{c}1 \\
20\end{array}$ & $\begin{array}{l}4 \\
5\end{array}$ & 6 \\
\hline Q17. Speak \& listen, & 4 & 1 & 13 & 2 & 17 & 1 & 9 & 1 & 2 & 1 & 4 & 6 \\
\hline
\end{tabular}




\begin{tabular}{|c|c|c|c|c|c|c|c|c|c|c|c|c|}
\hline \multirow{3}{*}{\begin{tabular}{|l|} 
Items \\
Frequency \\
Row \%
\end{tabular}} & \multicolumn{10}{|c|}{ Agreement rating } & \multirow{2}{*}{\multicolumn{2}{|c|}{ Total }} \\
\hline & \multicolumn{2}{|c|}{ Disagree++ } & \multicolumn{2}{|c|}{ Disagree } & \multicolumn{2}{|c|}{ Neutral } & \multicolumn{2}{|c|}{ Agree } & \multicolumn{2}{|c|}{ Agree++ } & & \\
\hline & : & ๘ & : & ৫ & : & $\underset{\sim}{\mathscr{S}}$ & : & $\mathbb{\leftrightarrow}$ & : & 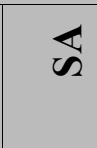 & : & $\underset{\infty}{\mathbb{s}}$ \\
\hline $\begin{array}{l}\text { precedence, read } \\
\text { \&write. }\end{array}$ & 8.9 & $\begin{array}{c}16 . \\
7\end{array}$ & $\begin{array}{c}28 . \\
9\end{array}$ & 33.3 & $\begin{array}{c}37 . \\
8\end{array}$ & $\begin{array}{c}16 . \\
7\end{array}$ & 20 & $\begin{array}{l}16 . \\
7\end{array}$ & 4.4 & $\begin{array}{c}16 . \\
7\end{array}$ & 5 & \\
\hline $\begin{array}{l}\text { Q18. Most of the time: } \\
\text { explain grammar }\end{array}$ & $\begin{array}{c}13 \\
28.9\end{array}$ & $\begin{array}{c}2 \\
33.3\end{array}$ & $\begin{array}{c}13 \\
28.9\end{array}$ & $\begin{array}{c}3 \\
50\end{array}$ & $\begin{array}{c}18 \\
40\end{array}$ & $\begin{array}{c}1 \\
16.7\end{array}$ & $\begin{array}{l}1 \\
2.2\end{array}$ & $\begin{array}{l}0 \\
0\end{array}$ & $\begin{array}{l}0 \\
0\end{array}$ & $\begin{array}{l}0 \\
0\end{array}$ & 45 & 6 \\
\hline $\begin{array}{l}\text { Q19. Necessary to teach } \\
\text { Chinese characters? }\end{array}$ & $\begin{array}{c}3 \\
6.5\end{array}$ & $\begin{array}{l}0 \\
0\end{array}$ & $\begin{array}{c}6 \\
13.0\end{array}$ & $\begin{array}{c}1 \\
20\end{array}$ & $\begin{array}{c}11 \\
23.9\end{array}$ & $\begin{array}{c}1 \\
20\end{array}$ & $\begin{array}{l}14 \\
30.4\end{array}$ & $\begin{array}{l}2 \\
40\end{array}$ & $\begin{array}{c}12 \\
26.1\end{array}$ & $\begin{array}{c}1 \\
20\end{array}$ & 46 & 5 \\
\hline $\begin{array}{l}\text { Q20. Use multimedia a } \\
\text { lot. }\end{array}$ & $\begin{array}{c}3 \\
6.5\end{array}$ & $\begin{array}{c}1 \\
16.7\end{array}$ & $\begin{array}{c}4 \\
8.7\end{array}$ & $\begin{array}{c}1 \\
16.7\end{array}$ & $\begin{array}{c}11 \\
23.9\end{array}$ & $\begin{array}{c}1 \\
16.7\end{array}$ & $\begin{array}{l}19 \\
41.3\end{array}$ & $\begin{array}{l}2 \\
33.3\end{array}$ & $\begin{array}{c}9 \\
19.6\end{array}$ & $\begin{array}{c}1 \\
16.7\end{array}$ & 46 & 6 \\
\hline $\begin{array}{l}\text { Q21. Teach both } \\
\text { minority language \& } \\
\text { Mandarin in the class. }\end{array}$ & $\begin{array}{l}27 \\
60\end{array}$ & $\begin{array}{c}6 \\
100\end{array}$ & $\begin{array}{c}14 \\
31.1\end{array}$ & $\begin{array}{l}0 \\
0\end{array}$ & \begin{tabular}{|c|}
2 \\
4.4
\end{tabular} & $\begin{array}{l}0 \\
0\end{array}$ & $\begin{array}{l}0 \\
0\end{array}$ & $\begin{array}{l}0 \\
0\end{array}$ & $\begin{array}{c}2 \\
4.4\end{array}$ & $\begin{array}{l}0 \\
0\end{array}$ & 45 & 6 \\
\hline $\begin{array}{l}\text { Q22. Cantonese is used } \\
\text { in CFL class. }\end{array}$ & $\begin{array}{c}25 \\
56.8 \\
\end{array}$ & $\begin{array}{c}5 \\
83.3 \\
\end{array}$ & $\begin{array}{c}17 \\
38.6 \\
\end{array}$ & $\begin{array}{c}1 \\
16.7 \\
\end{array}$ & $\begin{array}{c}1 \\
2.3\end{array}$ & $\begin{array}{l}0 \\
0\end{array}$ & $\begin{array}{l}0 \\
0\end{array}$ & $\begin{array}{l}0 \\
0\end{array}$ & $\begin{array}{c}1 \\
2.3\end{array}$ & $\begin{array}{l}0 \\
0\end{array}$ & 44 & 6 \\
\hline $\begin{array}{l}\text { Q23. Expect students to } \\
\text { adapt to teaching } \\
\text { methods. }\end{array}$ & $\begin{array}{c}10 \\
22.7\end{array}$ & $\begin{array}{c}3 \\
50\end{array}$ & $\begin{array}{c}9 \\
20.5\end{array}$ & $\begin{array}{c}2 \\
33.3\end{array}$ & $\begin{array}{c}21 \\
47.7\end{array}$ & $\begin{array}{l}0 \\
0\end{array}$ & $\begin{array}{l}4 \\
9.1\end{array}$ & \begin{tabular}{|l}
1 \\
16.7
\end{tabular} & $\begin{array}{l}0 \\
0\end{array}$ & $\begin{array}{l}0 \\
0\end{array}$ & 44 & 6 \\
\hline
\end{tabular}

Table 3 shows that CFL lecturers in the two countries have very similar teaching methods. They were satisfied (71.1\% in China, $66.7 \%$ in South Africa) with the ratio of lecturers to students. They agreed that memorisation is essential in CFL study $(80.4 \%$ in China; $83.3 \%$ in South Africa) and that students are the focus of CFL teaching (87\% in China; $66.7 \%$ in South Africa). Respondents agreed that CFL study is based on textbooks (76\% in China; $83 \%$ in South Africa) and CFL classes in both countries (68\% in China; $60 \%$ in South Africa) provide the opportunity to communicate and practise and cover four skills: speaking, listening, reading and writing. The CFL lecturers in the two countries agreed that practice is more important than explanation (73.4\% in China; $66.7 \%$ in South Africa). The lecturers in both countries agreed $(56.5 \%$ in China; $60 \%$ in South Africa) that it was necessary to teach Chinese characters from the beginning and all of them used multimedia a lot $(60.9 \%$ in China; 50 in South Africa). They disagreed that minority languages such as Cantonese are taught in CFL classes (91.1\% in China; $100 \%$ in South Africa). They do not spend most of the time in class to explain grammar $(57.8 \%$ in China; $83.3 \%$ in South Africa). In addition, they do not expect students to adapt to their teaching methods and they do not always use the same teaching method (65.9\% in China; $100 \%$ in South Africa).

However, lecturers in the two countries had a different perspective regarding teaching methods. Most respondents in China (60.8\%) agreed or strongly agreed that the traditional lecture method is good for CFL teaching but half of the respondents in South Africa gave a neutral response. Most respondents in China (84.8\%) agreed or strongly agreed that Chinese is the only language used in teaching CFL, while most respondents in South Africa disagreed (66.7\%). The respondents in China disagreed or strongly disagreed (60.5\%) that both Chinese and English should be used in teaching CFL, while $66.7 \%$ of respondents in South Africa 
agreed or strongly agreed to the use of both languages in tuition.

A possible reason for the similarities between CFL teaching methods in both countries is that most CFL lecturers in South Africa (66.7\%) come from China and they are seconded by Hanban for only one or two years' teaching. These teachers implement the pedagogy of their home country because they were trained in China. However, the background of students and the social language environment make the situation in South Africa different. China provides a dominant language setting in which Chinese is the mother tongue of the majority of the population and is used in all domains of everyday life: home, education, government, law, commerce and the media (Siegel, 2007). In this context, the expectations of students and teachers are that tuition will take place in the target language (Wang \& Lemmer, 2014). This constitutes an immersion programme in which the target language is the medium of classroom instruction (Baker, 2007). Contrary to this, Chinese is a small spoken language in South Africa and students, with low levels of Chinese proficiency, expect English to be used as the primary medium of instruction. In this case, the target language is the subject of formal study, but the medium of instruction is the L1 of all (or most) learners (Baker, 2007).

\section{Section E: The CFL curriculum}

Table 4 indicates respondent perceptions of the curriculum attributes according to country. In Table 4, column 1 refers to the number of questions; column 2 indicates the statistical significance of dependency per country; column 3 presents the statistical signification variable-level trend between countries; and column 4 provides the interpretation thereof. The observation NS stands for 'No Statistical Significance.'

Table 4 Summary of comparison of frequency distribution differences on CFL curriculum, section E, according to country

\begin{tabular}{|c|c|c|c|}
\hline Question & $\begin{array}{l}\text { Statistical } \\
\text { sign. of } \\
\text { dependen } \\
\text { cy with } \\
\text { country }\end{array}$ & $\begin{array}{c}\text { Statistic } \\
\text { al sign. } \\
\text { Variable } \\
- \\
\text { level } \\
\text { trend } \\
\text { between } \\
\text { countrie } \\
\text { s }\end{array}$ & Interpretation \\
\hline $\begin{array}{l}\text { Q2. Number } \\
\text { of courses } \\
\text { taught }\end{array}$ & NS & NS & $\begin{array}{l}\text { In both countries, most respondents teach } 2-3 \mathrm{CFL} \\
\text { courses. }\end{array}$ \\
\hline $\begin{array}{l}\text { Q3. Average } \\
\text { weekly } \\
\text { teaching } \\
\text { hours }\end{array}$ & NS & NS & $\begin{array}{l}\text { In both countries, most respondents teach } 11-20 \\
\text { hours per week. }\end{array}$ \\
\hline $\begin{array}{l}\text { Q4. Average } \\
\text { preparation } \\
\text { time }\end{array}$ & NS & NS & $\begin{array}{l}\text { In both countries, most respondents spend, on } \\
\text { average, between } 11 \text { and } 20 \text { hours per week } \\
\text { preparing CFL lessons. }\end{array}$ \\
\hline Q5. Typical & $* * *$ & $* * *$ & Statistically significant difference between countries \\
\hline
\end{tabular}




\begin{tabular}{|c|c|c|c|}
\hline $\begin{array}{l}\text { lesson } \\
\text { timetabling }\end{array}$ & & & $\begin{array}{l}\text { in the typical CFL lesson timetabling: in China, } \\
\text { lessons are presented mostly in the mornings; in SA, } \\
\text { lessons are presented at any time of the day. }\end{array}$ \\
\hline $\begin{array}{l}\text { Q6. } \\
\text { Additional } \\
\text { tutoring time } \\
\text { per week }\end{array}$ & $*$ & $* *$ & $\begin{array}{l}\text { Statistically significant difference between countries } \\
\text { in the additional tutoring time offered outside of } \\
\text { formal tuition: in South Africa lecturers spend more } \\
\text { time per week in additional tutoring than do Chinese } \\
\text { lecturers. }\end{array}$ \\
\hline $\begin{array}{l}\text { Q7. } \\
\text { Workload of } \\
\text { CFL } \\
\text { lecturers }\end{array}$ & NS & NS & $\begin{array}{l}\text { In both countries, respondent workload is 'ample' or } \\
\text { 'too much work'. }\end{array}$ \\
\hline $\begin{array}{l}\text { Q8. Most } \\
\text { important } \\
\text { element for } \\
\text { student } \\
\text { success }\end{array}$ & NS & $*$ & $\begin{array}{l}\text { Statistically significant difference between countries } \\
\text { in what is regarded as the most important success } \\
\text { element: in China, it is the language environment; in } \\
\text { SA, the number of hours students devote to study. }\end{array}$ \\
\hline $\begin{array}{l}\text { Q9. } \\
\text { Homework } \\
\text { routine }\end{array}$ & $* *$ & $* *$ & $\begin{array}{l}\text { Statistically significant difference between countries } \\
\text { in homework given: Chinese lecturers give } \\
\text { homework more regularly than SA lecturers do. }\end{array}$ \\
\hline $\begin{array}{l}\text { Q11. } \\
\text { Suitability of } \\
\text { textbooks }\end{array}$ & NS & NS & $\begin{array}{l}\text { In both countries, respondents indicate that textbooks } \\
\text { require some modification. }\end{array}$ \\
\hline $\begin{array}{l}\text { Q13. } \\
\text { Frequency of } \\
\text { textbook } \\
\text { revision }\end{array}$ & NS & $*$ & $\begin{array}{l}\text { Statistically significant difference between countries } \\
\text { in frequency of textbook revision: in China, } \\
\text { textbooks are revised much more regularly than in } \\
\text { SA. }\end{array}$ \\
\hline $\begin{array}{l}\text { Q14. } \\
\text { Lecturer } \\
\text { input on } \\
\text { design of } \\
\text { study } \\
\text { material }\end{array}$ & NS & NS & $\begin{array}{l}\text { In both countries, lecturers have to accept pre- } \\
\text { compiled study material. }\end{array}$ \\
\hline $\begin{array}{l}\text { Q16. CFL } \\
\text { pass rate }\end{array}$ & NS & NS & $\begin{array}{l}\text { Although the data seem to suggest that the Chinese } \\
\text { pass rate is higher than the South African pass rate, } \\
\text { no difference in pass rate patterns between the two } \\
\text { countries was indicated. }\end{array}$ \\
\hline \multicolumn{4}{|c|}{$\begin{array}{l}\text { Significance legend: } \\
\text { NS: no significance } \\
* \quad: \text { statistical significance on the } 5 \% \text { level of significance } \\
* *: \text { statistical significance on the } 1 \% \text { level of significance } \\
* * * \text { : statistical significance on the } 0.1 \% \text { level of significance }\end{array}$} \\
\hline
\end{tabular}

Table 4 indicates no statistical difference regarding the CFL curriculum in the two countries with reference to: the number of courses taught per lecture; the number of teaching hours per week; the average preparation time required for effective CFL instruction; the workload of CFL lecturers; the need for modification of textbooks; the use of pre-compiled study material; 
or the pass rate. In other words, it can be deduced that the everyday duties of delivering the CFL curriculum are very similar for lecturers in both countries.

However, Table 4 indicates statistically significant differences on the following aspects: typical lesson timetabling; time given to formal tutoring outside of classroom tuition per week; the most important element for student success; homework; and the frequency of textbook revision. These are important differences and are further explored in Tables 5 and 6 .

Table 5 Frequency distribution comparison on typical lesson timetabling according to country

\begin{tabular}{|l|c|c|c|}
\hline \multicolumn{1}{|c|}{ Typical CFL teaching timetabling } & Country & Total \\
\hline Morning & 40 & 1 & 41 \\
\hline Afternoon & 86.96 & 16.67 & \\
\hline At any time of the day & 1 & 0 & 1 \\
Total & 2.17 & 0.00 & 10 \\
Fisher's exact probability associated with the Pearson's chi-square test statistic of 17.95 is $<$ \\
Probability associated with the Cochran-Armitage trend test statistic of -4.16 is $<0.001 * * *$ \\
\hline
\end{tabular}

Table 5 indicates that, in the Chinese institutions, CFL lessons were presented mostly in the mornings $(86.96 \%)$ and in the South African institutions at any time of the day $(83.33 \%)$. A possible explanation is that CFL learning is the most important goal for CFL students in China. Consequently, most of the CFL lessons are presented in the morning. In South Africa, Chinese is an elective subject for CFL students, so it is presented at any time.

Table 6 Frequency distribution comparison of hours' additional tutoring time per week according to country

\begin{tabular}{|c|c|c|c|}
\hline \multirow[t]{2}{*}{ Additional tuition time per week } & \multicolumn{2}{|c|}{ Country } & \multirow[t]{2}{*}{ Total } \\
\hline & China & SA & \\
\hline None & $\begin{array}{c}32 \\
69.57\end{array}$ & $\begin{array}{c}1 \\
16.67\end{array}$ & 33 \\
\hline 1-3 hours & $\begin{array}{c}10 \\
21.74\end{array}$ & $\begin{array}{c}3 \\
50.00\end{array}$ & 13 \\
\hline 4-6 hours & $\begin{array}{c}4 \\
8.70\end{array}$ & $\begin{array}{c}2 \\
33.33\end{array}$ & 6 \\
\hline Total & 46 & 6 & 52 \\
\hline
\end{tabular}

Table 6 indicates that lecturers in South African institutions spend more time per week in additional tutoring outside of formal classroom time ( $50 \%$ of the respondents reported giving 
1-3 hours of tutoring per week, outside of classroom time). Chinese respondents indicated far less time being devoted to additional tutoring outside of formal classroom time. Seventy per cent $(69.57 \%)$ indicated that no time at all was spent on this task. A possible explanation is that China is a target-language-dominant environment (Siegel, 2007: 141), so CFL students have enough opportunity to practise Chinese after class. South Africa, however, is a firstlanguage-dominant environment (Siegel, 2007: 141), so CFL students do not have much opportunity to practise after class and they need the assistance of their lecturers. In addition, most Chinese respondents $(80 \%)$ felt that the most important element of success in CFL study was the immersion in the language environment. Although half of the South African participants agreed with the importance of a CFL-rich environment, a significant percentage $(33 \%)$ also indicated the hours devoted to study by students as being very important. A possible explanation is that China is a full-immersion language environment and so has the language environment advantage over South Africa. Chinese lecturers give homework more regularly than South African lecturers do: $52.17 \%$ of Chinese lecturers indicated that they gave homework every second lesson, while, in contrast, most respondents in South Africa (80\%) gave homework weekly. A possible explanation is that CFL students are full-time students of CFL in China, so they should do more work for CFL. However, CFL is an elective subject in South Africa, so the CFL lectures weigh lightly compared to students' other subjects in respect of doing homework. Another important reason could be that lecturers in China are stricter with students and place a heavier burden on them than do lecturers in South Africa. Chinese respondents observed that textbooks were revised much more regularly in China than in South Africa: $89 \%$ of Chinese respondents indicated an annual revision of textbooks. However, only half of respondents in South Africa (50\%) had observed the annual revision of textbooks. A possible explanation is that CFL teaching is very important in China and lecturers are concerned about the textbooks they use. In contrast, CFL is not a common elective course in South Africa and the textbooks used for CFL in South Africa are generally from China. Resources are not available to revise textbooks frequently.

\section{DISCUSSION AND CONCLUSION}

This article has presented the differences and similarities in CFL instruction as perceived by lecturers in universities in China and South Africa gathered through the survey according to five aspects: biographical profile, institutional factor, CFL students, teaching method and curriculum. In terms of staff employment, CFL in South Africa is a new and growing programme in higher education (cf. the low numbers of the staff) and supported by the Hanban of China (except Unisa). Fixed-term employee contract is typical of employment in South Africa, since most lecturers have been dispatched by China. However, permanent employment of lecturers can be expected to grow as CFL enrolment becomes stable in South Africa. In terms of institutional factors, CFL has a long history in China and thus significant differences are found between the two countries in terms of institutional infrastructure and support. As a growing enterprise, CFL provision in South African universities still has a long way to go. In terms of language environment, China has a strong target-language environment and CFL students come from various parts of the world with different language backgrounds. Chinese is the medium of instruction in the classroom and the medium of communication outside the classroom in China. Extra tutorial time for CFL oral practice is not as necessary as in South Africa, as students are immersed in Chinese in everyday life and have ample opportunities for oral practice. In contrast, in South Africa, the medium of communication in and outside the classroom is English. Therefore, tutorial time is very important for CFL students to practise oral communication, but it is limited. In terms of 
programme design, CFL study is the main aim for CFL students in China so the courses occur in the mornings and study hours are much longer than that of other courses. In South Africa, Chinese is an elective foreign language course, so the time schedule and the study hours are the same as other foreign language courses.

In conclusion, this article presented the findings of comparative study of teaching CFL at universities in China and in South Africa through a survey of lecturers' perceptions of tuition in both countries' contexts. The differences and similarities illustrated in this article have covered five aspects of the lecturers' experience: biographical profile, institutional factors, perceptions of CFL students, teaching methods and curriculum. The lecturers in China held statistically significantly more positive perceptions on the issues of institutional support, perceptions of CFL students, teaching methods and curriculum than lecturers in South Africa. This can be explained partly by the different linguistic contexts: CFL tuition in China takes place in a dominant-language environment where learning is reinforced both in and outside the classroom. In South Africa, language teaching and learning are primarily limited to the formal setting.

\section{REFERENCES}

ARTHUR, J, M WARING, R COE \& L HEDGES. 2012. Research Methods \& Methodologies in Education. Thousand Oaks: Sage.

BAKER, C. (2007). Bilingual Education: An Introductory Reader. Clevedon: Multilingual Matters.

BBC NEWS. 2006a. How hard is it to learn Chinese? Available from http://news.bbc.co.uk/hi/uk/_news/magazine/4617646.stm [Accessed: 6 June 2009]

CONFUCIUS INSTITUTE ANNUAL DEVELOPMENT REPORT. 2013. Available from http://english.hanban.org/ [Accessed: 3 November 2014]

CONFUCIUS INSTITUTE DEVELOPMENT PLAN. 2012-2020. Available from http://www.hanban.edu.cn/news/article/2013-02/28/content_486140.htm [Accessed: 15 August 2013]

HANBAN. 2011. Confucius Institute headquarters. Available from http://english.hanban.org/ node_7716.htm [Accessed: 12 August 2011]

HAU-YOON, L. 2002. The evaluation of Mandarin Chinese courses taught as a foreign language for distance learners. Unpublished MEd dissertation, University of South Africa, Pretoria.

JOHNSON, AP. 2008. A short guide to action research. Boston: Allyn \& Bacon.

KRAMSCH, C. 1994. Context and culture in language teaching. Oxford: Oxford University Press.

KOSHY, V. 2005. Action research for improving practice: A practical guide. London: Paul Chapman.

LU, J. \& ZHAO, Y. 2011. Teaching Chinese as a foreign language in China: A profile, in Teaching and learning Chinese in global contexts, edited by L. Tsung \& $\mathrm{K}$. Cruickshank. London: Continuum International Publishing Group.

MCMILLAN, JH \& S SCHUMACHER. 2006. Research in education: Evidence-based inquiry. Boston: Pearson Education.

MERTERNS, DM. 2010. Research and evaluation in education and psychology ( $\left.3^{\text {rd }} \mathrm{ed}\right)$. Thousand Oaks: Sage.

MINISTRY OF EDUCATION. 2002. Institute of Applied Linguistics. Available from 
www.china-language.gov.cn [Accessed: 6 June 2009]

MINISTRY OF EDUCATION OF CHINA. 2006. A brief introduction of teaching Chinese as a foreign language (TCFL) of China. Available at: http://www.Ministryof Education. The People's Republic of China.htm [Accessed: 7 June 2009]

RHODES UNIVERSITY. 2012. CIRU and Affiliates. Available from http://www.ru.ac.za/confuciusinstitute/ciruandaffiliates/ [Accessed: 25 March 2013]

RHODES UNIVERSITY. 2014. Course Outline. Available from http://www.ru.ac.za/confuciusinstitute/courseoutlines/ [Accessed: 5 November 2014]

SIEGEL, J. 2007. Social Context, in Handbook of Second Language Acquisition. In Doughty, CJ \& M. H. Long (Eds). Oxford: Blackwell Publishing.

STELLENBOSCH UNIVERSITY. 2009. Available from http://www.ccs.org.za/institute.html [Accessed: 22 June 2009]

STELLENBOSCH UNIVERSITY. 2011. Modern foreign languages. Available from http://www.sun.ac.za/forlang/ [Accessed: 23 August 2011]

UNIVERSITY OF CAPE TOWN. 2013. Available from

http://www.confucius.uct.ac.za/about/welcome/ [Accessed: 6 February 2013]

UN OFFICIAL LANGUAGES. 2013. Available from http://www.ealmanac.com/1798/numbers/the-six-official-languages-of-the-unitednations/. [Accessed 23 May 2013]

UNIVERSITY OF SOUTH AFRICA. 2011a. Mandarin Chinese. Available from http://www.unisa.ac.za/default.asp?Cmd= ViewContent\&ContentID=1784 [Accessed: 15 August 2011]

UNIVERSITY OF SOUTH AFRICA. 2011b. Centre for Asian Business. Available from www.unisa.edu.au/asianbusiness/news/default.asp [Accessed: 25 October 2011]

UNIVERSITY OF SOUTH AFRICA. 2013. Mandarin Chinese. Available from http://www.unisa.ac.za/_cmsys/default.asp? Cmd=ViewContent\&ContentID=7563 [Accessed: 27 March 2013]

USA TODAY. 2007. Chinese economy slows to still sizzling 11.5\% growth. Available from www.usatoday.com/money/.../2007-10-25-china-gdp_N.htm [Accessed: 5 June 2009]

WANG QI, Y \& EM LEMMER. 2013. Teaching Mandarin as a foreign language in higher education institutions in South Africa. Per Linguam, 29(1):33-48.

WANG QI, Y \& EM LEMMER. 2014. Worlds part: experiences of students learning Chinese as foreign language in universities in China and South Africa. Mediterranean Journal of Social Sciences. 5(15):414-423.

WANG, Y. (2013). A Comparative study of Mandarin as a Foreign Language in China and South Africa at Higher Education. Unpublished doctoral thesis. Pretoria, University of South Africa.

ZHAO, W. 2012. Personal communication and telephonic interview with Ms W Zhao, senior lecturer in Mandarin. 21 May 2012.

\section{Biographical note}

Yuhua Wang is an Associate Professor at the School of International Education, Tianjin Polytechnic University, China.

Email address: 2490328574@qq.com

EM Lemmer is a Professor at the College of Education, University of South Africa. Email address: lemmeem@unisa.ac.za 